\title{
Flux reversal in a simple random-walk model on a fluctuating symmetric lattice
}

\author{
Jesús Casado-Pascual \\ Física Teórica, Universidad de Sevilla, Apartado de Correos 1065, Sevilla 41080, Spain \\ (Received 15 November 2005; revised manuscript received 11 March 2006; published 11 August 2006)

\begin{abstract}
A rather simple random-walk model on a one-dimensional lattice is put forward. The lattice as a whole switches randomly between two possible states which are spatially symmetric. Both lattice states are identical, but translated by one site with respect to each other, and consist of infinite arrays of absorbing sites separated by two nonabsorbing sites. Exact explicit expressions for the long-time velocity and the effective diffusion coefficient are obtained and discussed. In particular, it is shown that the direction of the steady motion can be reversed by conveniently varying the values of either the mean residence times in the lattice states or the transition rates to the absorbing and nonabsorbing sites.
\end{abstract}

DOI: 10.1103/PhysRevE.74.021112

PACS number(s): 05.40.Fb, 05.60.Cd, 87.16.Nn

\section{INTRODUCTION}

The study of the transport of Brownian particles along periodic structures has attracted considerable and ever growing interest in the past years due to its relevance in a wide variety of problems in physics, chemistry, biology, and nanotechnology $[1,2]$. As required by the second law of thermodynamics, Brownian motion cannot induce a steady directed motion in a system at equilibrium (see, for instance, Ref. [1] for a detailed proof). Nevertheless, a Brownian particle can display a directed motion in a periodic potential which switches randomly or periodically between two or more states [3-7]. For this to be possible, certain spatiotemporal symmetry and supersymmetry conditions must be broken [8]. An important phenomenon often observed in these systems is the reversal of the current direction upon variation of a system parameter $[9,10]$. Though in many cases the spatiotemporal symmetry breaking is achieved by using a spatially asymmetric periodic potential, in Ref. [11] it was shown that it can also be obtained by using a spatially symmetric periodic potential which fluctuates between three states, one of them being of free diffusion. As shown in Ref. [12], if the symmetry of glide reflection is broken, a directed transport can also be induced in a spatially symmetric potential which switches randomly between two states. Besides the purely theoretical interest, the study of this kind of models is relevant because it may provide valuable information about the workings of the so-called molecular motors $[1,13]$. Molecular motors are able to travel along polymer filaments by utilizing chemical energy from ATP (adenosine triphosphate) hydrolysis, and are responsible for the transport of different substances inside the eukaryotic cells.

Recently, it has been demonstrated experimentally that the Brownian motion of a colloidal sphere can be rectified using a periodic potential that alternates between three [14] or two [15] states which differ only by a discrete translation. Each state is spatially symmetric and consists of a large array of optical traps, created with dynamic holographic optical tweezers, each of which can localize a colloidal sphere. The pattern of discrete optical traps can be modeled as a periodic array of Gaussian wells of width $\sigma$ and depth $V_{0}$, uniformly separated by a distance $L$ substantially larger than $\sigma$. In the three-state case considered in Ref. [14], the initial array of optical traps is extinguished after time $T$ and replaced immediately with a second array, which is displaced from the first by $L / 3$. This process is repeated after time $T$ with an additional displacement of $L / 3$. A cycle is completed when, after time $T$, the array of traps is displaced once again by $L / 3$ and returns to its initial state. In the two-state case reported in Ref. [15], the first step is the same as in [14], i.e., the initial array of optical traps is extinguished after time $T_{1}$ and replaced immediately with a second array, which is displaced from the first by $L / 3$. This second array is extinguished after time $T_{2}$ and replaced again by the first, thereby completing one cycle. One main result of the three-state case is that the direction of the motion depends sensitively on the duration of the states, $T$, relative to the time required for the particle to diffuse the intertrap separation [14]. By contrast, in the two-state case this direction depends on the relative durations of the two states $T_{2} / T_{1}$ [15]. Specifically, the flux is directed from each optical trap in the longer-duration state toward the nearest optical trap in the short-lived state, and vanishes for $T_{1}=T_{2}$. Thus, in both cases, by varying the value of the corresponding parameter the flux can be reversed.

To gain a deeper insight into the behavior of these systems, it would be desirable to develop simplified models, amenable to analytical treatment, which capture the key features of the original complex systems. The main focus of this paper is to present such a simplified model for the experiment described in Ref. [15]. The paper is organized as follows. In the following section, we introduce the model and provide definitions of the quantities of interest, namely, the long-time velocity and the effective diffusion coefficient. In Sec. III, we obtain explicit expressions for these two quantities. The main results arising from these expressions are discussed in Sec. IV. Finally, we present conclusions for the main findings of our work.

\section{DESCRIPTION OF THE MODEL}

In our simplified model the motion of the colloidal sphere in the periodic potential is modeled by a random walk on a one-dimensional lattice with its sites located at $x_{n}=n h$, with $n \in \mathbb{Z}$, where it has been assumed that the distance between two consecutive sites $x_{n+1}-x_{n}=h$ is independent of $n$. The lattice can be in two possible states that will be indicated by 


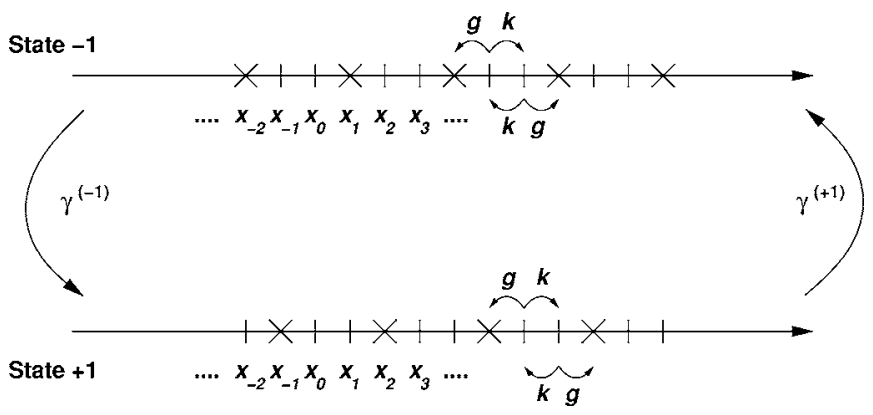

FIG. 1. Sketch of the fluctuating lattice. The absorbing sites are depicted by crosses.

the label $\alpha= \pm 1$. In contrast with the approach described in Ref. [15] in which the potential switches periodically between its two states, here we will assume that the lattice fluctuates randomly between its two possible states. The evolution of the joint probabilities $p_{n}^{(\alpha)}(t)$ that the particle is at site $x_{n}$ and the lattice in state $\alpha$ at time $t$ are governed by the master equation

$$
\begin{aligned}
\dot{p}_{n}^{(\alpha)}(t)= & -\left[L_{n}^{(\alpha)}+R_{n}^{(\alpha)}\right] p_{n}^{(\alpha)}(t)+R_{n-1}^{(\alpha)} p_{n-1}^{(\alpha)}(t)+L_{n+1}^{(\alpha)} p_{n+1}^{(\alpha)}(t) \\
& +\gamma^{(-\alpha)} p_{n}^{(-\alpha)}(t)-\gamma^{(\alpha)} p_{n}^{(\alpha)}(t)
\end{aligned}
$$

where $\gamma^{(\alpha)}$ is the transition rate from the lattice state $\alpha$ to $-\alpha$, and $L_{n}^{(\alpha)}$ and $R_{n}^{(\alpha)}$ are the transition rates from site $x_{n}$ to site $x_{n-1}$ and $x_{n+1}$, respectively, assuming that the lattice is in state $\alpha$. The initial condition is irrelevant since we are interested in the long-time behavior of the system.

Retaining the main features of the model described in Ref. [15], we will assume that both states of the lattice are spatially symmetric (i.e., there exists a $n^{\prime} \in \mathbb{Z}$ such that $L_{n^{\prime}+n}^{(\alpha)}$ $=R_{n^{\prime}-n}^{(\alpha)}$ for all $n \in \mathbb{Z}$ ) and periodic with period $N$ (i.e., $L_{n+N}^{(\alpha)}$ $=L_{n}^{(\alpha)}$ and $R_{n+N}^{(\alpha)}=R_{n}^{(\alpha)}$ for all $\left.n \in \mathbb{Z}\right)$. This is an important distinction between our model and other similar models previously reported in the literature [16-19], in which at least one of the lattice states is spatially asymmetric. For simplicity, we will restrict our study to the case $N=3$, which is the smallest value of the period for which a nonzero steady directed motion is possible under the above conditions of spatial symmetry and periodicity [20]. The lattice state $\alpha=+1$ is obtained from the lattice state $\alpha=-1$ by translating it to the right one site, so that, $L_{n}^{(+1)}=L_{n-1}^{(-1)}$ and $R_{n}^{(+1)}=R_{n-1}^{(-1)}$. When the lattice is in state $\alpha=-1$, there are absorbing sites at the locations $x_{3 j+1}$, for all $j \in \mathbb{Z}$, and consequently, $L_{3 j+1}^{(-1)}=R_{3 j+1}^{(-1)}$ $=L_{3 j+2}^{(+1)}=R_{3 j+2}^{(+1)}=0$. These absorbing sites play the role of the optical traps in our simplified model. The transition rates to an absorbing and a nonabsorbing site will be denoted by $g$ and $k$, respectively, so that $L_{3 j+2}^{(-1)}=R_{3 j}^{(-1)}=L_{3 j}^{(+1)}=R_{3 j+1}^{(+1)}=g$ and $R_{3 j+2}^{(-1)}=L_{3 j}^{(-1)}=R_{3 j}^{(+1)}=L_{3 j+1}^{(+1)}=k$. A sketch of the model just described is shown in Fig. 1.

It is worthwhile to point out that the model just described is formally equivalent to another random-walk model which mimics the motion of some molecular motors. In this model the walker switches randomly between two possible states, indicated by the label $\alpha= \pm 1, \gamma^{(\alpha)}$ being the transition rate from the state $\alpha$ to $-\alpha$ (see Fig. 2). These states are charac-

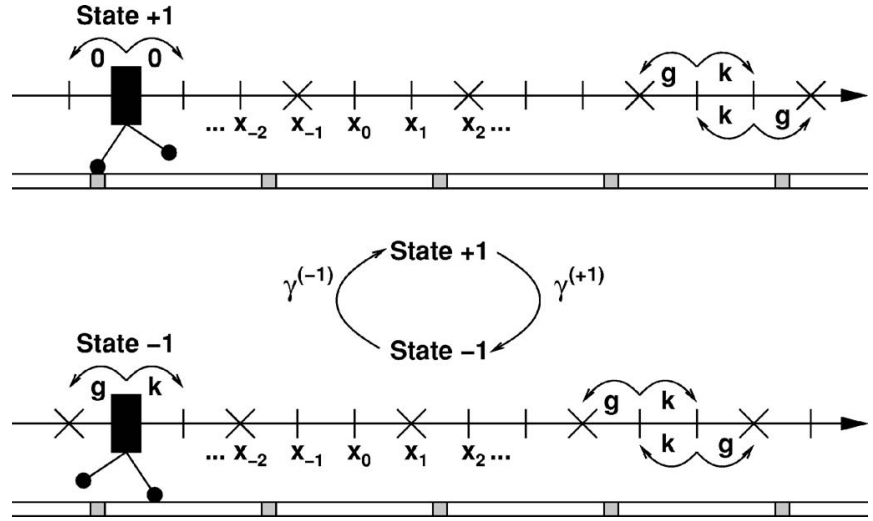

FIG. 2. An alternative interpretation of the model sketched in Fig. 1. The binding and absorbing sites are depicted by gray squares and crosses, respectively.

terized by the position of two legs, each with a head-shaped object attached at one of their ends. The walker "walks" on a rod which possesses an infinite array of binding sites, depicted by gray squares in Fig. 2. These binding sites are separated from each other by a distance $3 h$, and translated by $h / 2$ with respect to the sites of the lattice. Whenever any of the walker's "heads" comes in contact with a binding site, the walker stays trapped at that site until a fluctuation of its state occurs. For each state of the walker, the sites of the lattice with this property (absorbing sites) are depicted by crosses in Fig. 2. When none of the walker's heads are in contact with a binding site, the walker can hop to its nearestneighbor sites. Then, the transition rate from a site to its neighbors is $g$, if after hopping any of the heads comes in contact with a binding site, or $k$, otherwise. This interpretation of the model differs from the one considered at the end of Ref. [15], in three important aspects. First, ours is discrete and exactly solvable. Second, in ours the walker fluctuates randomly between its two possible states, whereas in theirs it switches periodically between them. In this sense, our model may be more effective for describing real molecular motors, since in this class of molecules the directed motion arises as a consequence of a competition between two stochastic time scales (the ATP binding rate and the ATP hydrolysis rate). Third, in [15] the direction of the steady motion depends only on the relative durations of the two states, whereas here, as we will see later on, it also depends on the sign of $g-k$.

We are interested in the study of the long-time velocity or flux $v$ and the effective diffusion coefficient $D_{\text {eff }}$, defined as

$$
v=\lim _{t \rightarrow \infty} \frac{d}{d t}\langle x(t)\rangle
$$

and

$$
D_{\text {eff }}=\frac{1}{2} \lim _{t \rightarrow \infty} \frac{d}{d t}\left[\left\langle x^{2}(t)\right\rangle-\langle x(t)\rangle^{2}\right],
$$

where the $l$ th moment of the position is given by $\left\langle x^{l}(t)\right\rangle$ $=h^{l} \sum_{\alpha= \pm 1} \sum_{n=-\infty}^{+\infty} n^{l} p_{n}^{(\alpha)}(t)$. 
There are certain properties of $v$ and $D_{\text {eff }}$ that can be predicted by simple considerations. For instance, in the absence of fluctuations between the two lattice states, both the long-time velocity and the effective diffusion coefficient will be zero, as the particle ends up being trapped by an absorbing site. Even if the sites depicted by crosses in Fig. 1 were not absorbing and, therefore, there were a nonzero transition rate from them (the same to the left and to the right), the long-time velocity would be also zero in the absence of fluctuations between the two lattice states. This is so because, in that case, both lattice states would be invariant under reflections about any of the sites with crosses. Another interesting property is obtained if one takes into account that the fluctuating periodic lattice sketched in Fig. 1 is invariant under the transformation $\{n, \alpha\} \Rightarrow\{-n,-\alpha\}$. Consequently, the longtime velocity and the effective diffusion coefficient fulfill the symmetry relations $v\left[\gamma^{(+1)}, \gamma^{(-1)}\right]=-v\left[\gamma^{(-1)}, \gamma^{(+1)}\right]$ and $D_{\text {efft }}\left[\gamma^{(+1)}, \gamma^{(-1)}\right]=D_{\text {efft }}\left[\gamma^{(-1)}, \gamma^{(+1)}\right]$, where we have written explicitly the dependence of $v$ and $D_{\text {eff }}$ on $\gamma^{(+1)}$ and $\gamma^{(-1)}$. Thus, if $\gamma^{(+1)}=\gamma^{(-1)}$, the long-time velocity will be zero.

\section{EXPLICIT EXPRESSIONS FOR THE LONG-TIME VELOCITY AND THE EFFECTIVE DIFFUSION COEFFICIENT}

In order to go further in the analysis $v$ and $D_{\text {eff }}$, it is necessary to obtain explicit expressions for them. To do that, we will extend the approach developed in Ref. [21] to the case of a fluctuating lattice. The procedure described here is quite general and can be applied to any fluctuating periodic lattice with an arbitrary period $N$. For this reason, in what follows it will be described in general terms. An alternative approach based on the gradient expansion of the probability distribution corresponding to a coarse-grained description of the random-walk model can be found in Ref. [17]. First, let us introduce the quantities

$$
q_{n}^{(\alpha)}(t)=\sum_{l=-\infty}^{\infty} p_{n+l N}^{(\alpha)}(t)
$$

and

$$
s_{n}^{(\alpha)}(t)=h \sum_{l=-\infty}^{\infty}(n+l N) p_{n+l N}^{(\alpha)}(t)-\langle x(t)\rangle q_{n}^{(\alpha)}(t),
$$

which are periodic in $n$ with period $N$, i.e., $q_{n+N}^{(\alpha)}(t)=q_{n}^{(\alpha)}(t)$ and $s_{n+N}^{(\alpha)}(t)=s_{n}^{(\alpha)}(t)$ for all $n \in \mathbb{Z}$. Substituting the expressions for the first and second moments of the position into Eqs. (2) and (3), and replacing $\dot{p}_{n}^{(\alpha)}(t)$ by the right-hand side of Eq. (1), it is not difficult to prove that the long-time velocity and the effective diffusion coefficient can be expressed as

$$
v=h \sum_{\alpha= \pm 1} \sum_{n=1}^{N}\left[R_{n}^{(\alpha)}-L_{n}^{(\alpha)}\right] Q_{n}^{(\alpha)}
$$

and
$D_{\text {eff }}=\frac{h^{2}}{2} \sum_{\alpha= \pm 1} \sum_{n=1}^{N}\left[R_{n}^{(\alpha)}+L_{n}^{(\alpha)}\right] Q_{n}^{(\alpha)}+h \sum_{\alpha= \pm 1} \sum_{n=1}^{N}\left[R_{n}^{(\alpha)}-L_{n}^{(\alpha)}\right] S_{n}^{(\alpha)}$,

where $Q_{n}^{(\alpha)}=\lim _{t \rightarrow \infty} q_{n}^{(\alpha)}(t)$ and $S_{n}^{(\alpha)}=\lim _{t \rightarrow \infty} S_{n}^{(\alpha)}(t)$. Thus, for the evaluation of $v$ and $D_{\text {eff }}$ it suffices to calculate the values of the $4 N$ numbers $Q_{n}^{(\alpha)}$ and $S_{n}^{(\alpha)}$, with $n=1, \ldots, N$ and $\alpha$ $= \pm 1$.

Differentiating Eqs. (4) and (5) with respect to time, making use of Eq. (1), as well as of the periodicity of $L_{n}^{(\alpha)}$ and $R_{n}^{(\alpha)}$, and taking into account that $\lim _{t \rightarrow \infty} \dot{q}_{n}^{(\alpha)}(t)$ $=\lim _{t \rightarrow \infty} \dot{s}_{n}^{(\alpha)}(t)=0$, it is easy to see that the long-time limits $Q_{n}^{(\alpha)}$ and $S_{n}^{(\alpha)}$ fulfill the set of $4 N$ algebraic equations

$$
\begin{gathered}
-\left[L_{n}^{(\alpha)}+R_{n}^{(\alpha)}\right] Q_{n}^{(\alpha)}+R_{n-1}^{(\alpha)} Q_{n-1}^{(\alpha)}+L_{n+1}^{(\alpha)} Q_{n+1}^{(\alpha)} \\
+\gamma^{(-\alpha)} Q_{n}^{(-\alpha)}-\gamma^{(\alpha)} Q_{n}^{(\alpha)}=0, \\
-\left[L_{n}^{(\alpha)}+R_{n}^{(\alpha)}\right] S_{n}^{(\alpha)}+R_{n-1}^{(\alpha)} S_{n-1}^{(\alpha)}+L_{n+1}^{(\alpha)} S_{n+1}^{(\alpha)} \\
+\gamma^{(-\alpha)} S_{n}^{(-\alpha)}-\gamma^{(\alpha)} S_{n}^{(\alpha)}=F_{n}^{(\alpha)},
\end{gathered}
$$

for $n=1, \ldots, N$ and $\alpha= \pm 1$, where

$$
F_{n}^{(\alpha)}=h\left[L_{n+1}^{(\alpha)} Q_{n+1}^{(\alpha)}-R_{n-1}^{(\alpha)} Q_{n-1}^{(\alpha)}\right]+v Q_{n}^{(\alpha)},
$$

and where the periodic boundary conditions $Q_{0}^{(\alpha)}=Q_{N}^{(\alpha)}$, $Q_{N+1}^{(\alpha)}=Q_{1}^{(\alpha)}, S_{0}^{(\alpha)}=S_{N}^{(\alpha)}$, and $S_{N+1}^{(\alpha)}=S_{1}^{(\alpha)}$ are assumed.

Summing up the $2 N$ equations contained either in the set of equations (8) or (9), it is easy to realize that in each set there are only $2 N-1$ independent equations (excluding some pathological cases where the number can be even lower). Thus, to univocally determine the $4 N$ quantities $Q_{n}^{(\alpha)}$ and $S_{n}^{(\alpha)}$, it is also necessary to add the two additional equations $\Sigma_{\alpha= \pm 1} \Sigma_{n=1}^{N} Q_{n}^{(\alpha)}=1$ and $\Sigma_{\alpha= \pm 1} \Sigma_{n=1}^{N} S_{n}^{(\alpha)}=0$, which can be straightforwardly proven from the definitions of $Q_{n}^{(\alpha)}$ and $S_{n}^{(\alpha)}$.

To apply the above procedure to the model sketched in Fig. 1, first one has to solve this set of equations, with $N$ $=3$ and the corresponding values for the transition rates $L_{n}^{(\alpha)}$ and $R_{n}^{(\alpha)}$. Replacing the results obtained for $Q_{n}^{(\alpha)}$ and $S_{n}^{(\alpha)}$ in Eqs. (6) and (7), one obtains after some lengthy simplifications that

$$
v=3 h \Delta \rho\left(1-\Delta \rho^{2}\right)(g-k) g k \Gamma^{2} A^{-1}
$$

and

$$
D_{\text {eff }}=\frac{9}{2} h^{2}\left(1-\Delta \rho^{2}\right) g k(g+k)(2 g+\Gamma) \Gamma A^{-1}-v^{2} B \Gamma^{-1} A^{-1},
$$

where

$$
\begin{gathered}
A=4 g^{2}(g+2 k)^{2}+8 g(g+k)(g+2 k) \Gamma \\
+\left[4(g+k)^{2}-\left(1+3 \Delta \rho^{2}\right) k^{2}\right] \Gamma^{2}, \\
B=4 g^{2}(g+2 k)^{2}+24 g(g+k)(g+2 k) \Gamma \\
+\left[28(g+k)^{2}-3\left(3+\Delta \rho^{2}\right) k^{2}\right] \Gamma^{2}+8(g+k) \Gamma^{3}, \quad(14) \\
\Gamma=\gamma^{(+1)}+\gamma^{(-1)}, \text { and } \Delta \rho=\rho_{\mathrm{eq}}^{(-1)}-\rho_{\mathrm{eq}}^{(+1)}, \rho_{\mathrm{eq}}^{(\alpha)} \text { being the equilib- }
\end{gathered}
$$


rium population of the lattice state $\alpha$, i.e., $\rho_{\mathrm{eq}}^{(\alpha)}=\gamma^{(-\alpha)} / \Gamma$. This quantity can alternatively be expressed as $\Delta \rho=\left[T^{(-1)}\right.$ $\left.-T^{(+1)}\right] /\left[T^{(-1)}+T^{(+1)}\right]$, where $T^{(\alpha)}=1 / \gamma^{(\alpha)}$ is the mean residence time of the lattice in the state $\alpha$.

\section{RESULTS}

An important result that follows from Eq. (11) is that the direction of the steady motion not only depends on the sign of $\Delta \rho$, as would be expected from the previous symmetry considerations, but also on the sign of the difference between the transition rates to an absorbing and a nonabsorbing site, $g-k$. Thus, for instance, if $T^{(-1)}>T^{(+1)}$, the steady directed motion is from left to right for $g>k$, whereas it is from right to left for $g<k$. Obviously, if $T^{(-1)}<T^{(+1)}$, the behavior is just the opposite. Finally, no steady directed motion is possible if either $T^{(-1)}=T^{(+1)}$ or $g=k$. This subtle dependence of the direction of the steady motion on the values of $g$ and $k$ cannot be inferred a priori from symmetry considerations, and is one of the main results of this work. It is important to point out that this model predicts correctly the direction of the steady motion of the experiment described in Ref. [15]. Indeed, in that case, the values of $g$ and $k$ can be estimated as the inverses of mean times required for the particle to diffuse a length $L / 3-\sigma / 2$ and $L / 3$, respectively, where $L$ is the intertrap distance and $\sigma$ is the width of an optical trap. Therefore, $g=2 D /(L / 3-\sigma / 2)^{2}$ and $k=2 D /(L / 3)^{2}, D$ being the diffusion coefficient of the colloidal sphere in the fluid. Since $g>k$, the motion is from left to right if $T^{(-1)}>T^{(+1)}$ and from right to left if $T^{(-1)}<T^{(+1)}$, i.e., from each absorbing site in the longer-duration state toward the nearest absorbing site in the short-lived state. The above expressions for $g$ and $k$ also show that the finite width of the optical traps plays an important role in the appearance of the steady directed motion.

Some limiting values of the expressions (11) and (12) can be easily interpreted. For instance, as suggested by the previous simple considerations, $v$ and $D_{\text {eff }}$ tend to zero both as $\Gamma \rightarrow 0$ (absence of fluctuations between the two lattice states) and as $\Delta \rho \rightarrow \pm 1$ (absence of fluctuations between the two lattice states in the long-time limit). As $g \rightarrow 0, v$ and $D_{\text {eff }}$ also go to zero because, in this limit, the particle stays trapped between two consecutive absorbing sites, since it is not allowed to jump to them. The expressions for $v$ and $D_{\text {eff }}$ also tend to zero both as $k \rightarrow 0$ and as $g \rightarrow \infty$. This is due to the fact that, in these limits, the motion of the particle consists of a series of forward and backward jumps from a nonabsorbing site to its nearest absorbing site, which arise as a consequence of the fluctuations in the lattice state. Finally, the limiting values $\lim _{\Gamma \rightarrow \infty} v=v_{\infty}$ and $\lim _{\Gamma \rightarrow \infty} D_{\text {eff }}=D_{\text {eff, } \infty}$, which can be easily calculated from Eqs. (11) and (12), also have a simple interpretation. Using the results in Ref. [21], it is not difficult to check that $v_{\infty}$ and $D_{\text {eff, } \infty}$ are the long-time velocity and the effective diffusion coefficient corresponding to a random walk with the average transition rates $L_{n}=\rho_{\mathrm{eq}}^{(+1)} L_{n}^{(+1)}$ $+\rho_{\text {eq }}^{(-1)} L_{n}^{(-1)}$ and $R_{n}=\rho_{\text {eq }}^{(+1)} R_{n}^{(+1)}+\rho_{\text {eq }}^{(-1)} R_{n}^{(-1)}$. In contrast with what happens in continuum models, the limiting value $v_{\infty}$ does not vanish, in general. This difference between discrete and continuum models has been previously pointed out in the literature (see, for example, Ref. [18]). From the expressions

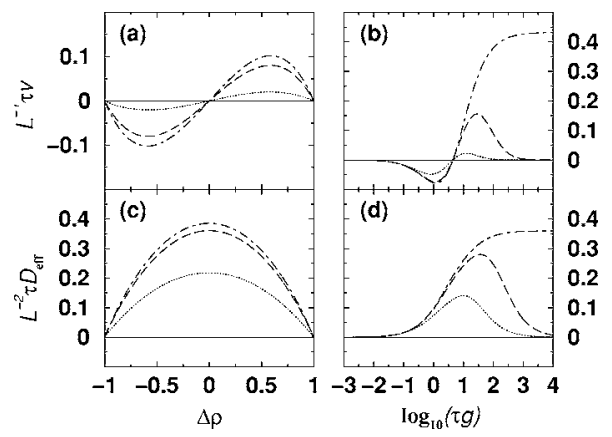

FIG. 3. Dependence of the dimensionless long-time velocity $L^{-1} \tau v$ (top panels) and effective diffusion coefficient $L^{-2} \tau D_{\text {eff }}$ (bottom panels) on $\Delta \rho$ (left panels) and $\log _{10}(\tau g)$ (right panels). The dimensionless parameter values are $L^{-1} h=\frac{1}{3}, \tau k=\frac{9}{2}, \tau g=9$ (left panels), $\Delta \rho=0.6$ (right panels), $\tau \Gamma=0$ (solid lines), $\tau \Gamma=10$ (dotted lines), and $\tau \Gamma=100$ (long-dashed lines). The limiting values $L^{-1} \tau v_{\infty}$ and $L^{-2} \tau D_{\text {eff, } \infty}$ are plotted with dash-dotted lines.

for $v_{\infty}$ and $D_{\text {eff, } \infty}$, it can be easily proved that $\lim _{g \rightarrow \infty} v_{\infty}$ $=3 h \Delta \rho\left(1-\Delta \rho^{2}\right) k / 4$ and $\lim _{g \rightarrow \infty} D_{\text {eff, } \infty}=9 h^{2}\left(1-\Delta \rho^{2}\right) k / 8$ which, in general, are not equal to zero. This reflects the fact that the limits $\Gamma \rightarrow \infty$ and $g \rightarrow \infty$ of Eqs. (11) and (12) do not commute.

In order to simplify the graphical presentation of results, it is convenient to introduce dimensionless quantities by defining characteristic length and time scales. The characteristic length scale $L$ is chosen to be the distance between two consecutive absorbing sites, i.e., $L=3 h$. The characteristic time scale $\tau$ is chosen to be the mean time required for the particle to diffuse the length $L$ in the absence of absorbing sites. Taking into account that in the absence of absorbing sites the effective diffusion coefficient is $D_{\text {eff,NA }}=h^{2} k$, it follows that $\tau=L^{2} /\left(2 D_{\text {eff,NA }}\right)=9 /(2 k)$. Then, the number of independent dimensionless parameters is reduced to only three (the values of $\tau \Gamma, \Delta \rho$, and $\tau g$ ), since the other two take the fixed values $L^{-1} h=\frac{1}{3}$ and $\tau k=\frac{9}{2}$.

In Fig. 3, we depict the dependence of the dimensionless long-time velocity $L^{-1} \tau v$ (top panels) and effective diffusion coefficient $L^{-2} \tau D_{\text {eff }}$ (bottom panels) on $\Delta \rho$ (left panels) and $\log _{10}(\tau g)$ (right panels). We have taken $\tau g=9$ (left panels), $\Delta \rho=0.6$ (right panels), and three values of $\tau \Gamma$, namely, $\tau \Gamma$ $=0$ (solid lines), 10 (dotted lines), and 100 (long-dashed lines). With dash-dotted lines we have plotted the limiting

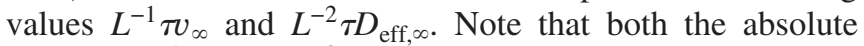
value of $L^{-1} \tau v$ and $L^{-2} \tau D_{\text {eff }}$ are increasing functions of $\tau \Gamma$, except at those points at which these functions vanish. This behavior is confirmed by a detailed study of the signs of the derivatives $\partial v / \partial \Gamma$ and $\partial D_{\text {eff }} / \partial \Gamma$. The reversal of the steady directed motion is clearly illustrated in panels (a) and (b). These panels also reveal that, for fixed values of the remaining parameters, there exist two optimal values of $\Delta \rho$ (opposite to each other) and of $\tau g$ which maximize the absolute value of the long-time velocity in each direction. In panel (b), one can also see that the location of the second maximum is shifted towards larger values of $\tau g$ as $\tau \Gamma$ is increased, and that this maximum completely disappears in the limit $\Gamma \rightarrow \infty$. Panel (c) shows that the dimensionless diffusion coefficient as a function of $\Delta \rho$ is maximized for $\Delta \rho=0$, i.e., 
when the mean residence times in each lattice state are equal. Analogously, there is also an optimal value of $\tau g$ which maximizes the dimensionless diffusion coefficient as a function of $\tau g$ [see panel (d)]. The location of this last maximum exhibits a similar behavior with $\tau \Gamma$ as the second maximum in panel (b).

\section{CONCLUSIONS}

In summary, we have proposed a simple random-walk model which allows the analytical study of the flux reversal in a spatially symmetric fluctuating lattice. We have obtained explicit analytical expressions for the long-time velocity and the effective diffusion coefficient and made a study of them. In spite of its simplicity, by appropriately choosing the transition rates to an absorbing and a nonabsorbing site, our model is able to reproduce some important features of the experiment described in Ref. [15]. In particular, it provides the correct direction of the induced flux and shows that this direction is closely related to the finite width of the optical traps. Furthermore, our model predicts the behavior of the effective diffusion coefficient that would be observed in the experiment reported in Ref. [15]. Finally, it predicts that similar results to those contained in [15] would be observed if the array of optical traps switched randomly between its two possible configurations instead of periodically.

We have also presented an alternative interpretation of our model which mimics the motion of some molecular motors. In contrast to the one considered at the end of Ref. [15], we have proven that, in ours, the direction of the steady motion depends not only on the relative durations of the two states, but also on the sign of the difference between the transition rates to an absorbing and a nonabsorbing site.

\section{ACKNOWLEDGMENTS}

The author acknowledges the support of the Ministerio de Educación y Ciencia of Spain (FIS2005-02884) and the Junta de Andalucía.
[1] P. Reimann, Phys. Rep. 361, 57 (2002).

[2] R. D. Astumian and P. Hänggi, Phys. Today 55(11), 33 (2002).

[3] M. Bier and R. D. Astumian, Phys. Rev. Lett. 76, 4277 (1996).

[4] J. Prost, J. F. Chauwin, L. Peliti, and A. Ajdari, Phys. Rev. Lett. 72, 2652 (1994).

[5] J. F. Chauwin, A. Ajdari, and J. Prost, Europhys. Lett. 27, 421 (1994); 32, 373 (1995).

[6] J. Rousselet, L. Salome, A. Ajdari, and J. Prost, Nature (London) 370, 446 (1994).

[7] L. P. Faucheux, L. S. Bourdieu, P. D. Kaplan, and A. J. Libchaber, Phys. Rev. Lett. 74, 1504 (1995).

[8] P. Reimann, Phys. Rev. Lett. 86, 4992 (2001).

[9] C. R. Doering, W. Horsthemke, and J. Riordan, Phys. Rev. Lett. 72, 2984 (1994).

[10] T. C. Elston and C. R. Doering, J. Stat. Phys. 83, 359 (1996).

[11] Y.-D. Chen, Phys. Rev. Lett. 79, 3117 (1997).

[12] R. Kanada and K. Sasaki, J. Phys. Soc. Jpn. 68, 3759 (1999).

[13] J. Howard, Mechanical of Motor Proteins and the Cytoskel- eton (Sinauer Associates, Sunderland 2001).

[14] S.-H. Lee, K. Ladavac, M. Polin, and D. G. Grier, Phys. Rev. Lett. 94, 110601 (2005).

[15] S.-H. Lee and D. G. Grier, Phys. Rev. E 71, 060102(R) (2005).

[16] A. B. Kolomeisky and B. Widom, J. Stat. Phys. 93, 633 (1998).

[17] J. A. Freund and L. Schimansky-Geier, Phys. Rev. E 60, 1304 (1999).

[18] H. Ambaye and K. W. Kehr. Physica A 267, 111 (1999).

[19] Y. A. Makhnovskii, V. M. Rozenbaum, D.-Y. Yang, S. H. Lin, and T. Y. Tsong, Phys. Rev. E 69, 021102 (2004).

[20] Indeed, if $N=2$, from the periodicity and the symmetry property it follows that $R_{1}^{(\alpha)}=L_{1}^{(\alpha)}$ and $R_{2}^{(\alpha)}=L_{2}^{(\alpha)}$. Consequently, from Eq. (6) one has that $v=0$. Analogously, if $N=1, v=0$ trivially.

[21] B. Derrida, J. Stat. Phys. 31, 433 (1983). 